\title{
Role of C-Jun-N-Terminal Kinase in Pregnane X Receptor-Mediated Induction of Human Cytochrome P4503A4 In Vitro
}

\author{
Guncha Taneja, ${ }^{1}$ Chun Chu, Paramahamsa Maturu, Bhagavatula Moorthy, and Romi Ghose ${ }^{2}$ \\ Department of Pharmacological and Pharmaceutical Sciences, College of Pharmacy, University of Houston (G.T., R.G.), and \\ Department of Pediatrics, Baylor College of Medicine (C.C., P.M., B.M.), Houston, Texas
}

Received November 7, 2017; accepted February 1, 2018

\begin{abstract}
Cytochrome P450 CYP3A4 is the most abundant drug-metabolizing enzyme and is responsible for the metabolism of $\sim 50 \%$ of clinically available drugs. Induction of CYP3A4 impacts the disposition of its substrates and leads to harmful clinical consequences, such as failure of therapy. To prevent such undesirable consequences, the molecular mechanisms of regulation of CYP3A4 need to be fully understood. CYP3A4 induction is regulated primarily by the xenobiotic nuclear receptor pregnane-X receptor (PXR). After ligand binding, PXR is translocated to the nucleus, where it binds to the CYP3A4 promoter and induces its gene expression. PXR function is modulated by phosphorylation(s) by multiple kinases. In this study, we determined the role of the c-Jun $\mathrm{N}$-terminal kinase (JNK) in PXR-mediated
\end{abstract}

\section{Introduction}

CYP3A4 enzyme is the most important contributor of hepatic and intestinal metabolism in adults (Krishna and Klotz, 1994; Guengerich, 1995; Leeder and Okey, 1996; Wilkinson, 2005). CYP3A4 is responsible for the metabolism of $\sim 50 \%$ of drugs currently used therapeutically for a wide spectrum of disorders, such as cancer, fungal and bacterial infections, neurologic disorders, hepatitis, AIDS, etc. (Guengerich, 1999; Veith et al., 2009; Zanger and Schwab, 2013). CYP3A4 also plays a crucial endogenous role in bile acid detoxification and metabolism of steroid hormones (Waxman et al., 1991; Araya and Wikvall, 1999).

Induction of CYP3A4 enzyme expression and activity alters the absorption, disposition, metabolism, and elimination of coadministered drugs (Thummel and Wilkinson, 1998). For example, supplementation

This work was supported by National Institutes of Health [Grant R21DA03575101A1 to R.G.], and [Grants R01 ES-019689, ES-001932, HL-129794, and HL-112516 to B.M.].

${ }^{1}$ Current affiliation: DILlsym Services, A Simulations Plus Company, Research Triangle Park, North Carolina.

${ }^{2}$ Current affiliation: Primary Laboratory of Origin.

This work was previously presented as Taneja G, Chu C, Moorthy B, and Ghose R (2016) C-Jun-N-terminal kinase (JNK): novel regulator of PXR-mediated CYP3A4 induction. ASPET-Experimental Biology, San Diego, CA, and Taneja G, Chu C, Moorthy B, and Ghose R (2015) Regulation of human hepatic CYP3A4 expression: role of cytokines and MAP-kinases at 2015 American Association of Pharmaceutical Scientists annual meeting and exposition, Orlando, FL.

https://doi.org/10.1124/dmd.117.079160. induction of CYP3A4 enzyme in vitro. Human liver carcinoma cells (HepG2) were transfected with CYP3A4 luciferase and PXR plasmids, followed by treatment with JNK inhibitor (SP600125; SP) and PXR activators rifampicin (RIF) or hyperforin. Our results indicate that SP treatment significantly attenuated PXR-mediated induction of CYP3A4 reporter activity, as well as gene expression and enzyme activity. JNK knockdown by siRNA (targeting both JNK 1 and 2) also attenuated CYP3A4 induction by RIF. Interestingly, SP treatment attenuated JNK activation by RIF. Furthermore, treatment with RIF increased PXR nuclear levels and binding to the CYP3A4 promoter; SP attenuated these effects. This study shows that JNK is a novel mechanistic regulator of CYP3A4 induction by PXR.

ABBREVIATIONS: AHR, aryl hydrocarbon receptor; ChIP, chromatin immunoprecipitation; DMSO, dimethylsulfoxide; ERK, extracellular signalregulated kinase; HepG2, human liver carcinoma cells; HRP, horseradish peroxidase; JNK, c-JUN N-terminal kinase; MAPK, mitogen-activated protein kinase; NR, nuclear receptor; P450, cytochrome P450; PCR, polymerase chain reaction; PXR, pregnane X receptor; RIF, rifampicin; VDR, vitamin D receptor. 
(Staudinger et al., 2011). So far, PXR phosphorylation has shown a repressive effect on the transcription of its target genes. PXR phosphorylation by cyclic AMP-dependent protein kinase A represses CYP3A4 gene expression in a species-specific manner (Ding and Staudinger, 2005a; Lichti-Kaiser et al., 2009b). Similarly, cyclindependent kinase (Cdk2 and 5) phosphorylates PXR to inhibit CYP3A4 expression. PXR phosphorylation by Cdk2 at Ser350 inhibits CYP3A4 gene expression in HepG2 cells (Lin et al., 2008). Activation of protein kinase $\mathrm{C}$ by proinflammatory cytokines inhibits PXR transcriptional activity in hepatocytes (Ding and Staudinger, 2005b). Furthermore, PXR phosphorylation at Thr57 by p70 S6K inhibited PXR activity (Pondugula et al., 2009a). Although direct phosphorylation of PXR is not involved, recent studies showed that casein kinase 2-mediated phosphorylation of heat shock protein 90 stabilizes PXR and induces its downstream genes (Kim et al., 2015). Heat shock protein 90 is a cytoplasmic retention protein that binds to and sequesters PXR in the cytosol (Squires et al., 2004). Interestingly, the mitogen-activated protein kinase (MAPK) c-Jun $\mathrm{N}$-terminal kinase (JNK) was required for optimal activation of CYP3A4 gene by 1,25 dihydroxyvitamin $\mathrm{D}_{3}\left(1,25(\mathrm{OH})_{2} \mathrm{D}_{3}\right)$, which is a known ligand for the NR, VDR (Rochel et al., 2000; Vanhooke et al., 2004; Yasunami et al., 2004). MAPKs consist of two additional members, extracellular signal-regulated kinase (ERK 1/2) and p38 kinase (Zhou et al., 2009). These serine/threonine kinases transduce extracellular signals from activated receptors on the cell surface to cellular responses by phosphorylating transcriptional factors or downstream kinases (Houliston et al., 2001).

The goal of this investigation was to determine the mechanistic role of JNK in CYP3A4 induction by PXR in vitro. We observed that inhibition of JNK significantly attenuated PXR-mediated induction of CYP3A4 reporter activity, gene expression, and enzyme activity in HepG2 cells. We also confirmed that CYP3A4 enzyme activity was attenuated by treatment of HepaRG cells with a JNK inhibitor. Furthermore, activation of JNK was observed upon treatment of HepG2 cells with the PXR ligand RIF. Interestingly, JNK inhibitors also attenuated ligand-induced PXR nuclear translocation and binding to the CYP3A4 promoter.

To date, PXR phosphorylation at multiple sites has inhibited PXR activity, leading to attenuation of CYP3A4 induction by PXR. To our knowledge, this is the first study showing that $\mathrm{JNK}$ is required for PXR-mediated CYP3A4 induction. This finding indicates that JNK may have a novel mechanistic role in CYP3A4 regulation by modulation of PXR function. These findings may provide insight into understanding functional interactions between cell signaling pathways and drug metabolism and their consequences in drug efficacy and/or toxicity.

\section{Materials and Methods}

Reagents and Materials. HepG2 cells were purchased from ATCC (Manassas, VA). HepaRG cells were purchased from Thermo Fisher Scientific (HPRGC10; Waltham, MA). RIF (3-4-methylpiperazinyliminomethyl) (R3501), hyperforin (dicyclohexylammonium) salt $\left(\mathrm{C}_{35} \mathrm{H}_{52} \mathrm{O}_{4} \cdot \mathrm{C}_{12} \mathrm{H}_{23} \mathrm{~N}\right)(\mathrm{H} 1792)$, curcumin (diferuloylmethane) (C1386), and dimethylsulfoxide (DMSO) were purchased from Sigma-Aldrich (St. Louis, MO). SP600125 (1, 9-pyrazoloanthrone) (tlrl-sp60), SB203580 (4-[4-(4fluorophenyl)-2-(4-methylsulfinylphenyl)-1H-imidazol-5-yl] pyridine) (tlrl-sb20), and PD098059 (2-(2-amino-3-methoxyphenyl) chromen-4-one) (tlrl-pd98) were purchased from InvivoGen (San Diego, CA). A custom siRNA sequence (AGAAUGUCCUACCUUCUUUUU) that simultaneously targets JNK1 and JNK2 and a control siRNA targeting luciferase were both synthesized by Dharmacon (Lafayette, CO). DharmaFECT Duo Transfection reagent was also purchased from Dharmacon. SuperFect Transfection Reagent was purchased from Qiagen (301305; Valencia, CA). p-CYP3A4-pGL3B luciferase plasmid was obtained as a gift from Dr. Rommel G. Tirona, The University of Western Ontario, London, ON). Human-PXR-pSG5 plasmid was obtained as a kind gift from Dr. Steven Kliewer, University of Texas Southwestern Medical Center, Dallas, TX. pRL-TK vector (E2241), Dual-Glo luciferase reporter assay kit (E1910), and P450-Glo CYP3A4 Assay (Luciferin-IPA) (V9002) was purchased from Promega (Madison, WI). Antibodies against phosphoJNK (9251), JNK (no. 9252), phospho-c-Jun (9164), and c-Jun (9165) were purchased from Cell Signaling (Beverly, MA), PXR (PA5-19080) was purchased from Thermo Scientific, and Lamin A/C (sc-20681) was purchased from Santa Cruz Biotechnology (Dallas, TX). Goat anti-rabbit IgG horseradish radish peroxidase (HRP) conjugate antibody (1706515) was purchased from Bio-Rad (Hercules, CA). Donkey anti-goat IgG-HRP (sc-2020) was purchased from Santa Cruz Biotechnology. Chromatin immunoprecipitation assay was performed using the Magna chromatin immunoprecipitation (ChIP) HiSens chromatin immunoprecipitation kit (17-10460) purchased from EMD-Millipore (Billerica, MA). Anti-PXR ChIP grade antibody (SC-25381X) was purchased from Santa Cruz Biotechnology.

Cell Culture and Transfection. HepG2 cells were maintained in Dulbecco's modified Eagle's medium (Life Technologies, Carlsbad, CA) supplemented with $10 \%$ fetal bovine serum (Atlanta Biologicals, Flowery Branch, GA). Briefly, cells were seeded in 96-well $\left(2.0 \times 10^{4}\right.$ cells/well $), 24$-well $\left(8.0 \times 10^{4}\right.$ cells/well $)$, or six-well format $\left(4.0 \times 10^{5}\right.$ cells/well $)$, depending on the type of assay at $80 \%$ confluency. After 24 hours, cells achieved $95 \%$ viability and were transfected with CYP3A4 luciferase (luc) promoter construct [contains the proximal promoter $(-362 /+53)$ and distal XREM (-7836/-7208; inserted in pGL3 Basic vector] and h-PXR-pSG5 plasmid together with the pRL-TK plasmid (which expresses Renilla luciferase) using SuperFect transfection reagent. For siRNA experiments, cotransfection of plasmids and siRNA was carried out with DharmaFect Duo reagent. A custom siRNA sequence targeting JNK 1 and JNK2 simultaneously and a control nontargeting siRNA were used for these experiments. HepG2 cells were transfected with JNK1/2 siRNA along with CYP3A4 luciferase and PXR plasmid using DharmaFect Duo reagent for 24 hours before treatment. HepaRG cells were cultured according to the manufacturer's protocol. Cryopreserved cells were thawed with Williams' E medium containing HepaRG thawing/plating medium supplement. Cells were seeded in 96-well plates $(100 \mu 1 /$ well $)$ at a density of 0.1 million cells $/ \mathrm{ml}$. The cells recovered at $37^{\circ} \mathrm{C}$ with a $5 \% \mathrm{CO}_{2}$ for 72 hours to achieve $\sim 90 \%$ viability.

Measurement of Luciferase Reporter Gene Expression. After 24 hours of transfection, HepG2 cells were pretreated with $30 \mu \mathrm{M}$ SP600125 (SP; specific JNK 1/2/3 inhibitor), $25 \mu \mathrm{M}$ curcumin (JNK pathway inhibitor), $10 \mu \mathrm{M}$ SB203580 (p-38 inhibitor), $25 \mu \mathrm{M}$ PD098059 (ERK inhibitor), or DMSO $(0.1 \%)$ as control for 30 minutes, followed by treatment with $1 \mu \mathrm{M}$ hyperforin or $10 \mu \mathrm{M}$ RIF or DMSO $(0.1 \%)$ as control for 24 hours. After treatment, cells were lysed and Dual-Glo luciferase assay carried out according to manufacturer's instructions (E1910; Promega). Briefly, Dual-Glo luciferase buffer containing Dual-Glo luciferase substrate was added to the lysed cells, and luciferase activity was recorded. Firefly luciferase activity was normalized to Renilla luciferase activity and compared among different treatments.

Quantitative Real-Time Polymerase Chain Reaction. HepG2 cells were seeded in six-well plates at $80 \%$ confluency. After 24 hours, at $\sim 95 \%$ viability, cells were pretreated with vehicle $(0.1 \%$ DMSO) or $30 \mu \mathrm{M} \mathrm{SP}$ for 30 minutes, followed by $10 \mu \mathrm{M}$ RIF for $0,2,4,6,8$, and 12 hours. At each time point, total RNA was isolated using TRIzol reagent according to the manufacturer's protocol (Sigma-Aldrich). cDNA was synthesized using the high-capacity reverse transcription kit from Applied Biosystems (Foster City, CA). Real-time polymerase chain reaction (PCR) was performed using an ABI PRISM 7300 Sequence Detection System instrument and software (Applied Biosystems) as described previously (Ghose et al., 2004, 2007; Shah et al., 2014). In short, each 25- $\mu$ 1 reaction mixture contained $50-100 \mathrm{ng}$ of cDNA, $300 \mathrm{nM}$ forward primer, $300 \mathrm{nM}$ reverse primer, $200 \mathrm{nM}$ fluorogenic probe, and $15 \mu \mathrm{l}$ of TaqMan Universal PCR Master Mix. PCR thermocycling parameters were $50^{\circ} \mathrm{C}$ for 2 minutes, $95^{\circ} \mathrm{C}$ for 10 minutes, and 40 cycles of $95^{\circ} \mathrm{C}$ for 15 seconds, and $60^{\circ} \mathrm{C}$ for 1 minute. We extrapolated the quantitative expression values from standard curves, and these values were normalized to GAPDH. Data are represented as relative gene expression (normalized to vehicle control).

P450-Glo Activity Assay. HepG2 and HepaRG cells were cultured on whitewalled, collagen-coated 96-well culture plates at a density of $1.0 \times 10^{4}$ cells/well. HepG2 and HepaRG cells were incubated for 24 and 72 hours, respectively, followed by pretreatment with DMSO $(0.1 \%)$ or $30 \mu \mathrm{M}$ SP for 30 minutes and treatment with $10 \mu \mathrm{M}$ RIF or DMSO $(0.1 \%)$. After treatment, cells were lysed, and luciferase enzymatic activity was measured using a P450-Glo commercial kit according to the manufacturer's protocol (V9002; Promega). Specific CYP3A4 luminogenic substrate, luciferin-IPA, was added to the wells at a concentration of 

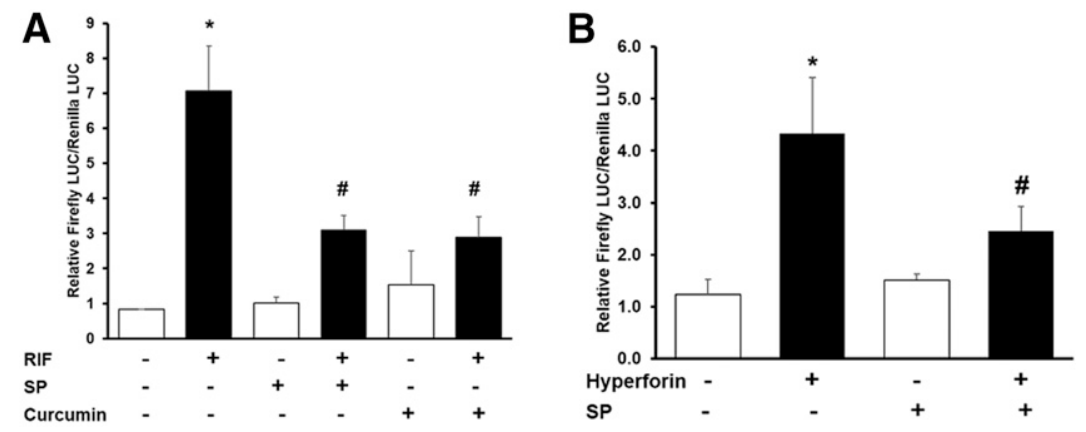

Fig. 1. Induction of CYP3A4-luciferase activity by RIF was attenuated by JNK inhibitors. CYP3A4 luciferase reporter was cotransfected with PXR plasmid into HepG2 cells. Cells were pretreated with vehicle (DMSO) or (A) $30 \mu \mathrm{M}$ SP/25 $\mu \mathrm{M}$ curcumin for 30 minutes, followed by $10 \mu \mathrm{M}$ RIF for 24 hours, or (B) $30 \mu \mathrm{M}$ SP for 30 minutes, followed by $2 \mu \mathrm{M}$ hyperforin for 48 hours and harvested for luciferase activity assays as described under Materials and Methods. The data are expressed as Firefly luciferase/Renilla luciferase activity and presented as the mean \pm S.D. from three independent experiments $(n=3)$ performed in triplicate. $* P<0.05$ compared with control treatment. ${ }^{\#} P<0.05$ compared with DMSO + RIF treatment.

$3 \mu \mathrm{M}$ and incubated at $37^{\circ} \mathrm{C}$ for 60 minutes. Light emission from the samples was detected by SpectraMax Microplate Reader/Luminometer, and net signal was calculated by subtracting background luminescence values.

Immunoblotting. HepG2 cells were seeded in six-well plates at $80 \%$ confluency. After 24 hours, at $\sim 95 \%$ viability, cells were pretreated with vehicle ( $0.1 \%$ DMSO) or $30 \mu \mathrm{M}$ SP for 30 minutes, followed by $10 \mu \mathrm{M}$ RIF. Whole-cell, nuclear, and cytosolic extracts were prepared as described previously (Ghose et al., 2007, 2011), and the protein concentrations were determined using the bicinchoninic acid (BCA) assay according to the manufacturer's protocol (Pierce, Rockford, IL). Immunoblotting was performed as described in previous publications (Moorthy et al., 2000; Jiang et al., 2004; Ghose et al., 2007, 2011). Briefly, equal amounts of protein $(30 \mu \mathrm{g})$ were analyzed by SDS-PAGE and transferred onto a nitrocellulose membrane. The membranes were incubated with phospho-JNK, JNK, phospho-c-Jun, c-Jun, PXR, and Lamin A/C primary antibodies overnight at $4{ }^{\circ} \mathrm{C}$, followed by incubation with goat anti-rabbit IgG-HRP secondary antibody or donkey anti-goat IgG secondary antibody for 60 minutes at room temperature. The membranes were then washed and incubated with HyGlo HRP antibody detection reagent (E2500; Denville Scientific, Hollister, MA) according to the manufacturer's instructions. The immunoreactive bands were detected by chemiluminescence method, and the band density was analyzed by Image J software (National Institutes of Health, Bethesda, MD). Band density of phospho-JNK 1 and 2 was measured and normalized to JNK 1 and 2 respectively. Phospho-cJun was normalized to c-jun bands. Total Lamin A/C was used as a loading control or as a housekeeping gene for nuclear extracts.

Chromatin Immunoprecipitation Assay. ChIP assays were performed to assess specific binding of the PXR complex to the PXR response element on the CYP3A4 promoter as described in the manufacturer's protocol (17-10460; EMD Millipore Inc.). HepG2 cells were seeded $\sim 80 \%-90 \%$ confluency $\left(1.0 \times 10^{7}\right.$ cells $/$ well $)$ in a $150-\mathrm{mm}$ culture dish containing $20 \mathrm{ml}$ of growth media. Next day, HepG2 cells were pretreated with SP $(30 \mu \mathrm{M})$ or DMSO $(0.1 \%)$ for 30 minutes, followed by RIF $(10 \mu \mathrm{M})$ for 24 hours. The cells were then crosslinked with $37 \%$ formaldehyde at room temperature for 10 minutes, washed twice with ice-cold phosphate-buffered saline, and collected in $1 \mathrm{ml}$ of ice-cold phosphate-buffered saline. Cells were pelleted at $800 \mathrm{~g}$ and digested by sonication (15 pulses, 20 seconds each, 30 seconds rest in between). The protein-DNA complexes were immunoprecipitated by using ChIP-grade anti-PXR antibody (SC-25381X; Santa Cruz). As a negative control, the beads were incubated with lysates without anti-PXR antibody. The chromatin was reverse crosslinked and eluted in $50 \mu 1$ of elution buffer. DNA recovered from this assay was analyzed by endpoint $\mathrm{PCR}$ using primers for $\mathrm{PXR}$ binding site in the $C Y P 3 A 4$ promoter region (forward 5'-AGAACCCAGAACCCTTGGAC-3' and reverse 5'-CTGCCTGCA GTTGGAAGAG- $3^{\prime}$ ). PCR products were analyzed by agarose gel electrophoresis. Ten percent of the total cell lysate was used as "input." Eluted DNA was further analyzed by real-time PCR using same primer mix as mentioned previously. ChIP DNA Ct values were normalized to input $\mathrm{Ct}$ values by subtracting the $\mathrm{Ct}$ value of the input (and dilution factor) from each sample according to method II of the manufacturer's protocol. Estimated fold enrichment of the positive locus sequence in ChIP DNA over negative control is expressed as fold enrichment or fold induction.

Statistical Analysis. All data are presented as the mean \pm S.D.s from at least three independent experiments $(n=3)$ unless otherwise specified. Differences between the groups were compared using Student's unpaired two-tailed $t$ test. One-way analysis of variance (ANOVA) with Dunnett's post hoc test was applied to the data if more than two groups were analyzed. $P$ value $<0.05$ was considered statistically significant.

\section{Results}

Induction of CYP3A4 Luciferase Activity by PXR Was Mediated by JNK. To determine the role of JNK in the induction of CYP3A4 reporter gene expression, we cotransfected HepG2 cells with plasmids expressing $C Y P 3 A 4$ reporter gene and PXR, followed by treatment with the PXR ligand RIF in the presence of JNK inhibitors. CYP3A4 reporter activity increased $\sim 8$-fold in the presence of $10 \mu \mathrm{M}$ RIF compared with control (Fig. 1A). This CYP3A4 reporter induction by RIF was significantly attenuated by treatment with SP, indicating that JNK may be involved in regulating CYP3A4 induction by PXR. We observed that PD098059 (ERK inhibitor) cotreatment also inhibited CYP3A4 luciferase activity but not SB203580 (p38 inhibitor) (data not shown). In addition, we studied the effect of curcumin, a JNK pathway inhibitor of CYP3A4 reporter expression. As shown in Fig. 1A, $15 \mu \mathrm{M}$ curcumin significantly attenuated RIF-mediated $C Y P 3 A 4$ induction by almost $50 \%$. A significant difference was not observed between curcumin treatment alone and curcumin with RIF treatment. We further confirmed these findings by treating HepG2 cells with another PXR ligand, hyperforin in the presence of the SP (Fig. 1B). Hyperforin is an active component of St. John's wort and a potent activator of human PXR (Moore et al., 2000; Chen et al., 2004). CYP3A4 luciferase activity was induced by $\sim 4$-fold by $2 \mu \mathrm{M}$ hyperforin at 24 hours, and this induction was significantly attenuated by SP treatment. SP had no effect on CYP3A4 luciferase activity in the absence of RIF or hyperforin (Fig. 1), indicating that JNK likely does not affect the basal expression of the CYP3A4 gene.

To confirm our results, we investigated the effect of simultaneous knockdown of JNK1 and JNK2 (using a siRNA that targets a homologous region present in both kinases) on CYP3A4 reporter gene induction (Fig. 2A). Knockdown of JNK1/2 expression reduced the ability of RIF to induce $C Y P 3 A 4$ luciferase activity in HepG2 cells, validating our previous data with pharmacologic inhibitors. No significant differences were found between control and JNK/siRNAtreated cells in the absence of RIF. The validity of the siRNAs was confirmed by the lack of JNK and phospho-JNK expression in JNK siRNA-treated cells (Fig. 2B). The fact that inhibition of JNK by pharmacologic inhibitors or siRNA attenuated CYP3A4 induction by two different PXR ligands (RIF and hyperforin) strongly indicates that PXR is likely regulated by JNK-dependent signaling mechanism. Furthermore, whereas CYP3A4 reporter expression in HepG2 cells without PXR plasmid was below the level of detection (data not shown), the same signal was significantly enhanced in PXR-transfected cells, suggesting a PXR-dependent effect.

Induction of CYP3A4 Gene Expression by PXR Was in Part Mediated by JNK. To determine the role of JNK in regulating endogenous $C Y P 3 A 4$ gene expression, HepG2 cells were treated with 


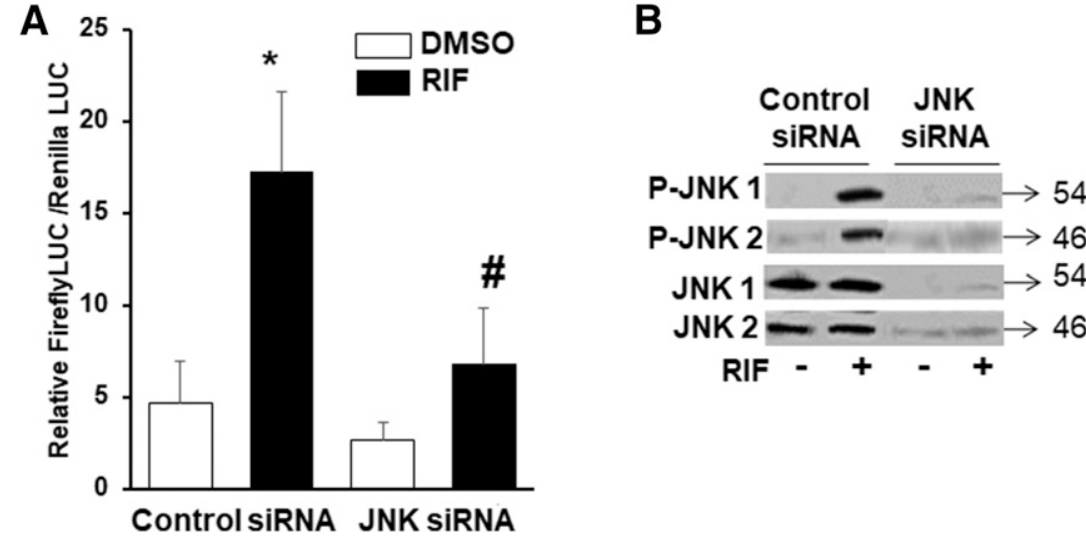

Fig. 2. Induction of $C Y P 3 A 4$ luciferase activity by RIF was attenuated by JNK siRNA. Custom-made JNK1/2 siRNA and control siRNA were cotransfected with CYP3A4 luciferase plasmid and PXR plasmid in HepG2 cells. After transfection, cells were treated with the vehicle (DMSO) or $10 \mu \mathrm{M}$ RIF. (A) Cells were lysed after 24 hours, and the data are expressed as Firefly luciferase/Renilla luciferase activity. The data are presented as the mean \pm S.D. from three independent experiments $(n=3)$ performed in triplicates. ${ }^{*} P<0.05$ as compared with vehicle control. ${ }^{\#} P<0.05$ as compared with RIFtreated control siRNA transfected cells. (B) Cells were lysed after 1 hour, and phospho (P)-JNK and JNK protein levels in wholecell extracts were determined by immunoblotting.
SP and RIF (Fig. 3). The cells were lysed to prepare mRNA, and CYP3A4 gene expression was analyzed. RIF treatment significantly induced $C Y P 3 A 4$ gene expression from 2 to 8 hours ( $\sim 3-4.5$ fold); the expression was lower at the 12-hour time point ( $\sim 2$-fold), although it was significantly induced compared with the control. We also observed 2-fold CYP3A4 mRNA induction by RIF at 24 hours (data not shown). SP treatment significantly attenuated this induction. About 2-fold CYP3A4 mRNA induction by RIF was observed in HepG2 cells by other groups at longer time points (Novotna and Dvorak, 2014). These results were in agreement with our $C Y P 3 A 4$ luciferase activity data in transfected HepG2 cells. Since significant attenuation of $C Y P 3 A 4$ reporter gene activity was observed with ERK inhibitor, we also carried out gene expression studies using the ERK inhibitor PD098059 and found that PD098059 had no effect on RIF-induced CYP3A4 mRNA levels (data not shown).

Induction of CYP3A4 Enzyme Activity by PXR was Mediated by JNK. We performed P450-Glo assay to determine the role of JNK in PXR-mediated induction of CYP3A4 enzyme activity. As shown in Fig. 4A, treatment of HepG2 cells with RIF significantly induced CYP3A4 enzyme activity ( 2.5 fold) compared with control, and SP attenuated this induction of CYP3A4 enzyme activity (as measured by the metabolism of the CYP3A4-specific substrate, luciferin IPA). To confirm our findings, we also conducted CYP3A4 activity assays in

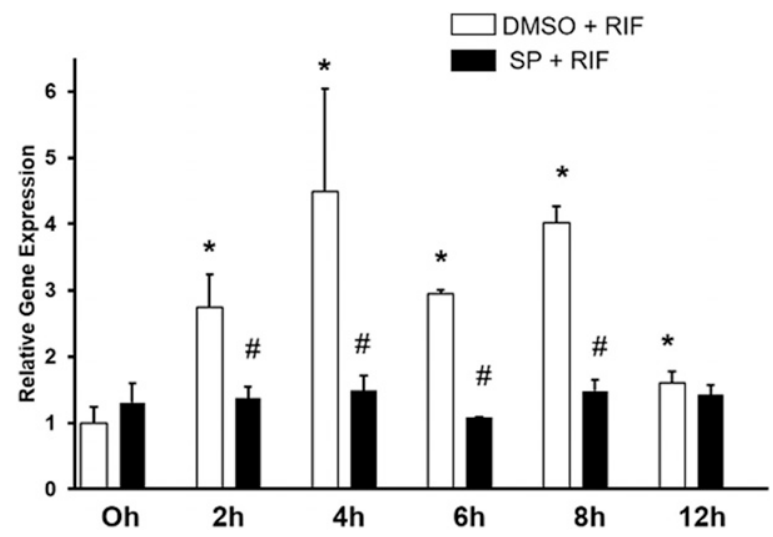

Fig. 3. Induction of $C Y P 3 A 4$ gene expression by RIF was attenuated by SP. HepG2 cells were pretreated with vehicle (DMSO) or $30 \mu \mathrm{M}$ SP for 30 minutes, followed by $10 \mu \mathrm{M}$ RIF for $0,2,4,6,8$, and 12 hours. Relative CYP3A4 mRNA expression (zero-hour samples are set as 1) levels are plotted at different times. The data are presented as the mean \pm S.D. from three independent experiments $(n=3)$ performed in triplicate and are expressed as the relative change normalized to vehicle treated cells. ${ }^{*} P<0.05$ compared with DMSO + RIF zero-hour group. ${ }^{\#} P<0.05$ as compared with DMSO + RIF for each time point.
HepaRG cells, which are terminally differentiated hepatic cells derived from a human hepatic progenitor cell line that retains many characteristics of primary human hepatocytes (Parent et al., 2004). We observed a similar trend in HepaRG cells; RIF treatment significantly induced CYP3A4 enzyme activity from 4 to 12 hours; this induction was attenuated by the JNK inhibitor, SP (Fig. 4B). In these cell lines, we did not see any changes in DMSO-treated cells over time, and SP by itself did not have any effect on CYP3A4 activity (data not shown). Consistent with our gene expression results, SP does not affect CYP3A4 enzyme activity in the absence of RIF, indicating that JNK is likely not involved in regulating basal CYP3A4 activity.

RIF Treatment Activated JNK In Vitro. Since treatment with JNK inhibitors resulted in attenuation of PXR-mediated CYP3A4 reporter activity, gene expression, as well as enzyme activity, we determined the role of RIF in activation of JNK. Whole-cell extracts were prepared from treated HepG2 cells, and immunoblot analysis was carried out to determine phospho-JNK 1 and 2 protein expression. JNK exists in three distinct isoforms (JNK1-3). Although JNK1 and JNK2 genes are ubiquitously expressed, including in the liver, the JNK3 gene is selectively expressed in the brain, heart, and testis (Ip and Davis, 1998). Both phospho-JNK1 (P-JNK1) and phospho-JNK2 (P-JNK2) levels were increased by RIF treatment starting at 60 minutes, although this increase was attenuated in the presence of the JNK inhibitor SP (Fig. 5). At 2 and 4 hours, p-JNK protein levels increased compared with zero-hour time point, but this effect was not significant. In agreement with these findings, we found that the JNK substrate, c-Jun was phosphorylated after RIF treatment of 4 hours, and this phosphorylation was attenuated in the presence of SP (Fig. 5, C and D).

PXR Nuclear Levels were Regulated by JNK. Our results indicate that JNK likely affects PXR function; therefore, we determined the role of JNK in regulating PXR nuclear levels in RIF-treated HepG2 cells. As expected, PXR nuclear protein levels significantly increased from 4 to 24 hours in the presence of RIF (Fig. 6). Importantly, SP treatment attenuated RIF-mediated nuclear accumulation of PXR, indicating that PXR protein levels in the nucleus are regulated by JNK.

PXR Binding to the CYP3A4 Promoter was Mediated by JNK. Lastly, we performed in vitro ChIP assays to analyze whether decreased accumulation of PXR in the nucleus resulting from JNK inhibition impacts the binding of PXR to the CYP3A4 promoter. A commercially available ChIP-grade anti-PXR antibody was used to precipitate DNAprotein complexes. RIF treatment alone significantly increased the association of PXR with the regulatory regions of the CYP3A4 gene compared with control. Both our endpoint and quantitative RT-PCR data showed decreased binding of PXR to CYP3A4 promoter when JNK is inhibited (Fig. 7), supporting the hypothesis that JNK was required for optimum binding of PXR to its response elements on CYP3A4 gene. We 
A

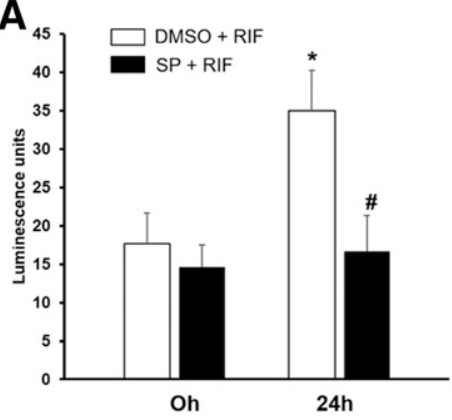

B

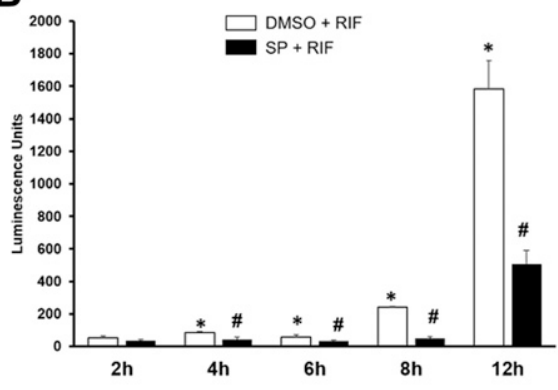

Fig. 4. Induction of RIF-mediated CYP3A4 activity was attenuated by SP. (A) HepG2 cells were pretreated with vehicle (DMSO) or $30 \mu \mathrm{M}$ SP for 30 minutes, followed by $10 \mu \mathrm{M}$ RIF for 24 hours. After treatment, cells were incubated with specific CYP3A4 substrate, $3 \mu \mathrm{M}$ Luciferin IPA for 60 minutes, and luminescence was detected. The data are presented as the mean \pm S.D. from three independent experiments $(n=3)$ performed in triplicate. $* P<0.05$ compared with DMSO + RIF zero-hour group. ${ }^{\#} P<0.05$ compared with DMSO + RIF 24 hours. (B) HepaRG cells were pretreated with vehicle (DMSO) or $30 \mu \mathrm{M}$ SP for 30 minutes, followed by $10 \mu \mathrm{M}$ RIF for 2, 4, 6, 8, and 12 hours. After treatment, cells were incubated with specific CYP3A4 substrate, $3 \mu \mathrm{M}$ Luciferin IPA for 60 minutes, and luminescence was detected. The data are presented as the mean \pm S.D. from three independent experiments $(n=3)$ performed in triplicate. $* P<0.05$ compared with DMSO + RIF 2-hour group. ${ }^{\#} P<0.05$ compared with DMSO + RIF for each time point. saw faint bands in cell lysates precipitated without anti-PXR antibody, which might be due to nonspecific binding. Input DNA from total cell lysate confirmed amplification of the $C Y P 3 A 4$ regulatory region targeted by specific primers.

\section{Discussion}

Induction of CYP3A4 enzyme expression/activity can have adverse effects on therapeutic outcome in patients. Therefore, the mechanism of CYP3A4 induction needs to be fully elucidated. This in vitro study demonstrates that CYP3A4 induction by PXR was mediated in part by a JNK-dependent mechanism. Research carried out in the last decade has shown that PXR-mediated gene expression is regulated not only by xenobiotics and endobiotics (Kliewer et al., 1998; Lehmann et al., 1998; Staudinger and Lichti, 2008; Pondugula et al., 2009b) but also by cellular signaling pathways. Post-translational modifications of PXR, especially direct phosphorylation, primarily led to an inhibition of its transcriptional activity (Wang et al., 2012); thereby downregulating Cyp3a expression. Our in vitro data, on the other hand, have shown that JNK is required for optimum induction of CYP3A4 via PXR. We provide additional evidence that PXR nuclear translocation. as well as binding of PXR to its response elements on CYP3A4 gene, is JNKdependent.

To understand comprehensively the role of MAPKs in PXR-mediated CYP3A4 signaling, we investigated the role of all MAPKs by treating HepG 2 cells with specific ERK 1/2 pathway inhibitor (PD098059), JNK 1/2/3 inhibitor (SP600125), and p38 inhibitor (SB203580). Specifically,
SP600125, an anthrapyrazolone compound, is a potent inhibitor of all isoforms of $\mathrm{JNK}$ with an $\mathrm{IC}_{50}$ of $0.04 \mu \mathrm{M}$, but it exhibits greater than 300-fold selectivity against other MAPKs- ERK and p38 ( $\left.\mathrm{IC}_{50}>10 \mu \mathrm{M}\right)$ (Bennett et al., 2001). In our studies, whereas JNK and ERK 1/2 inhibition significantly attenuated PXR-mediated CYP3A4 luciferase activity, p-38 inhibition showed no significant change. Curcumin, which can inhibit both JNK ( $\left.\mathrm{IC}_{50}: 5 \mu \mathrm{M}\right)$ and ERK $\left(\mathrm{IC}_{50}: 20 \mu \mathrm{M}\right)$ (Chen and Tan, 1998), also attenuated PXR-mediated CYP3A4 luciferase activity. MAPKs phosphorylate downstream kinases and nuclear factors, such as c-Jun, c-Fos, c-Myc, SP1, Elk1, etc. Since different MAPKs phosphorylate varying nuclear factors, it is possible that only nuclear factors activated by JNK and ERK might be involved in CYP3A4 regulation; however, the possibility of ERK being involved was eliminated from the results that the ERK inhibitor PD098059 had no effect on RIF-induced CYP3A4 mRNA expression in HepG2 cells (data not shown). A similar study by Yasunami et al. (2004) reported that the induction of CYP3A4 mRNA and promoter activity by the VDR ligand $1,25(\mathrm{OH})_{2} \mathrm{D}_{3}$ was attenuated in the presence of a JNK inhibitor, whereas ERK or p-38 inhibition had no effect. SP is one of the most widely used and extensively studied ATP-competitive JNK inhibitors (Bogoyevitch and Arthur, 2008; Davies and Tournier, 2012; Zhang et al., 2012; Feng et al., 2013; Koch et al., 2015), and curcumin has been shown to inhibit JNK signaling pathways (Chen and Tan, 1998; Suh et al., 2007); however, these compounds can target additional pathways to affect CYP3A4 expression. For example, both SP and curcumin can activate aryl hydrocarbon receptor (AHR) (Ciolino et al., 1998; Bachleda and Dvorák, 2008; Dvorak et al., 2008; Mohammadi-Bardbori et al., 2012); recent
A

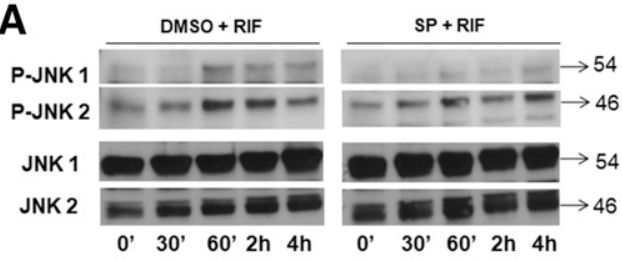

B

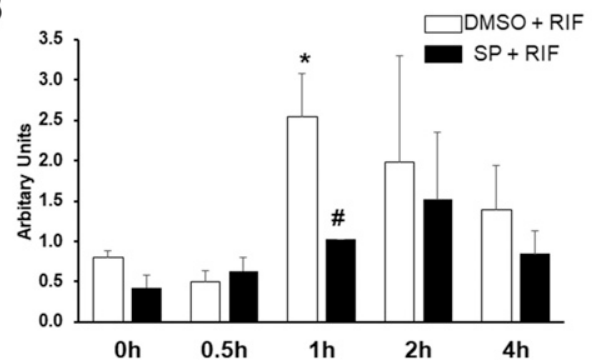

C
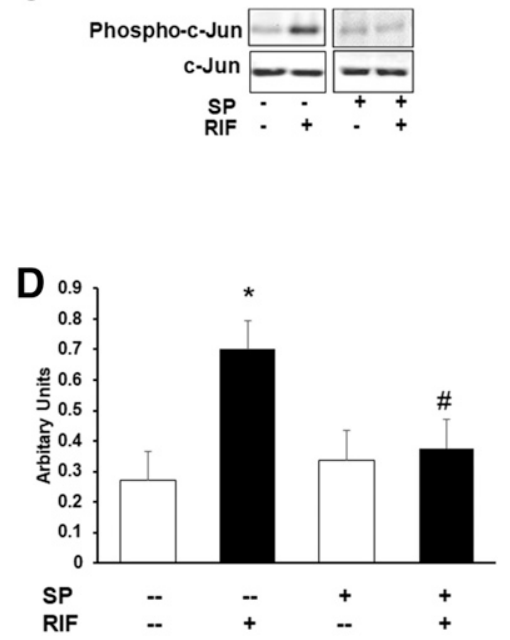

Fig. 5. RIF activates JNK in whole-cell extracts prepared from $\mathrm{HepG} 2$ cells. (A) Immunoblot of phospho (P)-JNK and JNK in whole-cell extracts after treatment of HepG2 cells with vehicle (DMSO) or $30 \mu \mathrm{M}$ SP for 30 minutes, followed by $10 \mu \mathrm{M}$ RIF for $0,0.5,1,2$, and 4 hours. (B) Representation of quantification of total $\mathrm{p}$-JNK (sum of p-JNK normalized levels) by densitometry after normalizing the P-JNK 1 and 2 levels over JNK 1 and 2, respectively. (C) Immunoblot of phospho-c-Jun in whole-cell extracts after treatment of HepG2 cells with vehicle (DMSO) or $30 \mu \mathrm{M}$ SP for 30 minutes, followed by $10 \mu \mathrm{M}$ RIF for 4 hours. The contrast was adjusted to improve legibility. (D) Representation of quantification of total phospho-c-Jun levels by densitometry after normalizing over total c-Jun. Replicates from three experiments performed in duplicate were quantified by densitometry. $* P<0.05$ compared with untreated control (DMSO) ${ }^{\#} P<0.05$ as compared with DMSO + RIF. 
A

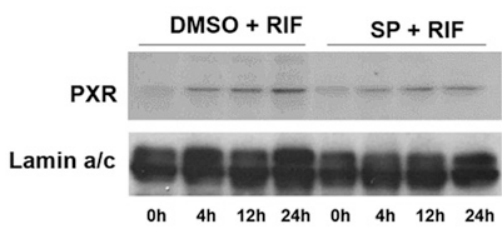

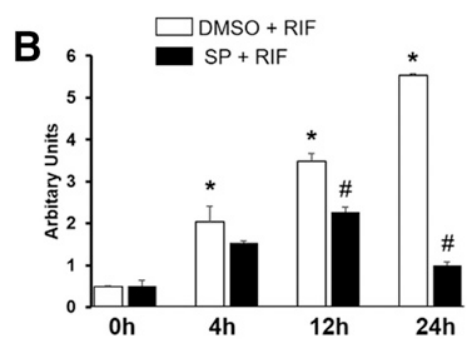

Fig. 6. Attenuation of RIF-induced PXR nuclear levels by SP. (A) Immunoblot of PXR in nuclear extracts after treatment of HepG2 cells with vehicle (DMSO) or $30 \mu \mathrm{M}$ SP for 30 minutes, followed by $10 \mu \mathrm{M}$ RIF for $0,4,12$, and 24 hours. (B) Quantification of blots by densitometry after normalizing PXR levels over total Lamin A/C nuclear housekeeping protein. Replicates from three experiments performed in duplicate were quantified by densitometry. ${ }^{*} P<0.05$ compared with DMSO + RIF zero-hour group. ${ }^{\#} P<0.05$ as compared with DMSO + RIF for each time point. studies have shown that AHR activation decreases basal and RIFinduced CYP3A4 expression in HepaRG cells (Rasmussen et al., 2017). So it is possible that SP or curcumin can activate AHR to reduce CYP3A4 expression; however, these compounds did not affect basal CYP3A4 expression in our experiments. To address the potential issues associated with nonspecific effects of the pharmacologic inhibitors, we confirmed our results by siRNA experiments. Knockdown of JNK1/2 expression by siRNA also attenuated $C Y P 3 A 4$ induction by RIF in HepG 2 cells. These data confirmed that JNK is indeed required for optimal activation of CYP3A4 as pharmacologic inhibition, as well as genetic knockdown of JNK1 and JNK2, attenuated its promoter activity significantly at 24 hours; however, the role of individual isoforms of JNK needs to be further investigated.

Additionally, we studied the effect of SP on RIF-mediated CYP3A4 mRNA and activity in HepG2 cells. HepG2 cells, are frequently used for in vitro biotransformation assays (Westerink and Schoonen, 2007). Although HepG2 is a cheap and easy-to-use cell line, endogenous activity and expression of enzymes and NRs is relatively low in HepG2 cells compared with primary human hepatocytes (Xu et al., 2004). Despite this limitation, HepG2 cells are routinely used for induction studies, and previous reports have shown significant induction of CYP3A4 gene expression ( $\sim 3$ - to 6-fold) as well as enzyme activity by RIF in these cells (Pascussi et al., 2001; Usui et al., 2003; Westerink and Schoonen, 2007). As an additional approach, we performed enzyme activity studies in HepaRG cells, which is a well established model for biotransformation applications (Gripon et al., 2002; Lambert et al., 2009; Anthérieu et al., 2010). In our data, attenuation of PXR-mediated induction of CYP3A4 enzyme activity in the presence of JNK inhibitor, irrespective of cell line, confirmed that JNK is required for induction of CYP3A4, and this is not a cell-line specific effect.

To understand the role of JNK in PXR regulation, we demonstrated that RIF activated JNK in HepG2 cells. Activation of PXR by RIF has previously been shown to increase phosphorylation of p38 MAPK; which was attributed to induction of GADD $45 \beta$ gene (Kodama and Negishi, 2011).
$G A D D 45 \beta$ is an immediate to early response gene induced by various physiologic and environmental stressors, including cytokines and genotoxic stresses (Liebermann and Hoffman, 2008). Our data showing activation of JNK by RIF in HepG 2 cells could potentially be mediated via similar targets of PXR. Alternatively, PXR may increase JNK expression; however, we did not detect any increase in total JNK protein levels in our experiments. Further studies to determine the mechanism of JNK activation by PXR are ongoing.

To determine the mechanism involved in JNK-mediated regulation of CYP3A4, we studied the role of JNK in regulating PXR nuclear translocation or its binding to CYP3A4 gene. PXR exists as a phosphoprotein in cells (Lichti-Kaiser et al., 2009a), and previous studies have shown that kinases decrease PXR transcription primarily either by strengthening PXR-corepressor interaction or weakening PXRcoactivator interactions. Recent studies have shown that activation of AMP-activated protein kinase and JNK by metformin lead to downregulation of PXR expression and reduced expression of carboxylesterases (Shan et al., 2017). In contrast, our studies showed that JNK positively regulated PXR function without affecting its expression (data not shown). JNK may positively regulate PXR function by multiple mechanisms (Fig. 8), including the following: 1) JNK directly phosphorylates PXR and increases its nuclear translocation/binding to the CYP3A4 gene; 2) JNK phosphorylates cytoplasmic retention proteins associated with PXR and impacts its translocation; 3) JNK decreases PXR-corepressor binding; or 4) JNK increases PXR-coactivator binding. Although we did not study whether PXR is directly phosphorylated by JNK, we found that JNK is required for PXR nuclear translocation and binding to the $C Y P 3 A 4$ promoter, ultimately affecting PXR function. Using in silico computer-based analysis using PhosphoSitePlus, we found nine serine phosphorylation sites and seven threonine phosphorylation sites on PXR, and further studies to reveal their association with JNK are ongoing. Similar to our study, seven serine/threonine residues were also identified in human PXR protein; these residues are good potential substrates for an array of kinases,
A

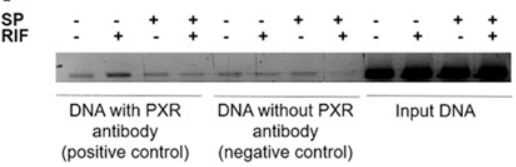

B

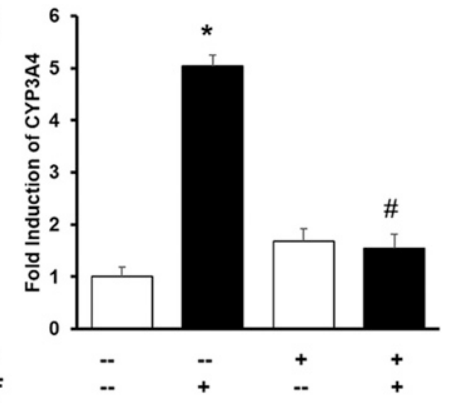

Fig. 7. Attenuation of binding of PXR to CYP3A4 promoter region by SP. HepG2 cells were pretreated with vehicle (DMSO) or $30 \mu \mathrm{M} \mathrm{SP}$ for 30 minutes, followed by $10 \mu \mathrm{M}$ RIF for 24 hours. An antibody against PXR was used to immunoprecipitate DNA-protein complexes. As a negative control, the beads were incubated with lysates without anti-PXR antibody. ChIP assays were performed as described under Material and Methods. (A) Endpoint PCR was performed using forward and reverse primers designed in the promoter region of CYP3A4 and analyzed on a $2 \%$ agarose gel. Ten percent of the total cell lysate was used as "input." (B) Quantitative real time-PCR was performed using forward and reverse primers designed in the promoter region of CYP3A4. Estimated fold enrichment of the positive locus sequence in ChIP DNA over negative control is expressed as fold enrichment or fold induction. Data represents mean of triplicates \pm S.D. from two independent experiments. $* P<0.05$ compared with control. ${ }^{\#} P<0.05$ compared with RIF treatment alone. 


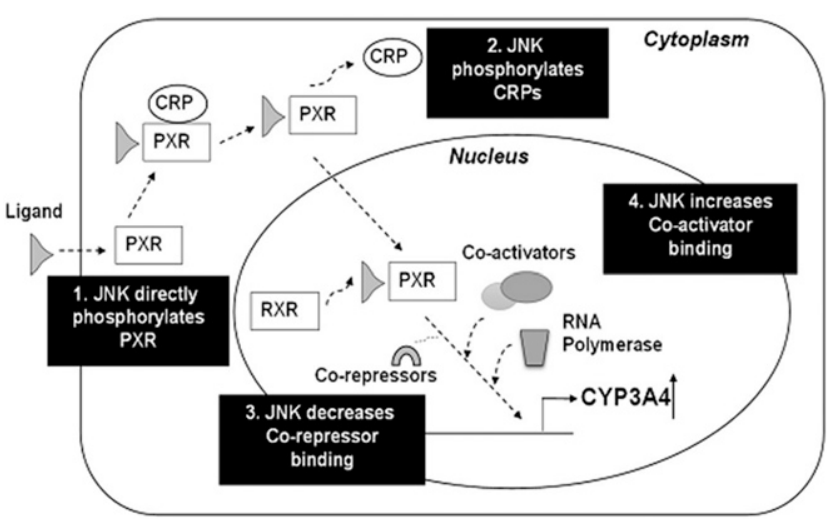

Fig. 8. Potential roles of JNK in PXR-mediated induction of CYP3A.

including MAPKs (Lichti-Kaiser et al., 2009a). Using mass spectrometry analysis, some of these serine-threonine residues-such as S114, T133/135, S167, and S200 in PXR-were also found to be phosphorylated by cyclin-dependent kinase 2 (Elias et al., 2014).

In summary, our in vitro data indicate that JNK, but not ERK or p38, is required for $C Y P 3 A 4$ gene activation by PXR. Further studies exploring the role of JNK on CYP3A4 induction in vivo will be clinically relevant in studying PXR-mediated target gene expression. JNK is activated by various extracellular stimuli and thus regulates gene expression through phosphorylation of transcription/nuclear factors. Hence, elucidation of the contribution of JNK in the xenobiotic-induced expression of P450 genes may be instrumental in understanding the mechanism of induction of $\mathrm{P} 450$ s, which can impact therapeutic outcome in patients undergoing treatment with multiple medications. Ultimately, understanding the role of JNK in induction of CYP3A4 could provide novel strategies to address concerns about loss of drug safety or efficacy resulting from alteration of expression and activity of the CYP3A4 enzyme.

\section{Authorship Contributions}

Participated in research design: Taneja, Moorthy, Ghose.

Conducted experiments: Taneja, Maturu, Chu.

Contributed new reagents or analytic tools: Moorthy, Ghose.

Performed data analysis: Taneja.

Wrote or contributed to the writing of the manuscript: Taneja, Moorthy, Ghose.

\section{References}

Anthérieu S, Chesné C, Li R, Camus S, Lahoz A, Picazo L, Turpeinen M, Tolonen A, Uusitalo J, Guguen-Guillouzo C, et al. (2010) Stable expression, activity, and inducibility of cytochromes P450 in differentiated HepaRG cells. Drug Metab Dispos 38:516-525.

Araya Z and Wikvall K (1999) 6 $\alpha$-Hydroxylation of taurochenodeoxycholic acid and lithocholic acid by CYP3A4 in human liver microsomes. Biochim Biophys Acta 1438:47-54

Bachleda P and Dvorák Z (2008) Pharmacological inhibitors of JNK and ERK kinases SP600125 and U0126 are not appropriate tools for studies of drug metabolism because they activate aryl hydrocarbon receptor. Gen Physiol Biophys 27:143-145.

Bennett BL, Sasaki DT, Murray BW, O'Leary EC, Sakata ST, Xu W, Leisten JC, Motiwala A, Pierce S, Satoh Y, et al. (2001) SP600125, an anthrapyrazolone inhibitor of Jun N-terminal kinase. Proc Natl Acad Sci USA 98:13681-13686.

Bogoyevitch MA and Arthur PG (2008) Inhibitors of c-Jun N-terminal kinases: JuNK no more? Biochim Biophys Acta 1784:76-93.

Chen Y, Ferguson SS, Negishi M, and Goldstein JA (2004) Induction of human CYP2C9 by rifampicin, hyperforin, and phenobarbital is mediated by the pregnane $\mathrm{X}$ receptor. J Pharmacol Exp Ther 308:495-501.

Chen YR and Tan TH (1998) Inhibition of the c-Jun N-terminal kinase (JNK) signaling pathway by curcumin. Oncogene 17:173-178.

Ciolino HP, Daschner PJ, Wang TT, and Yeh GC (1998) Effect of curcumin on the aryl hydrocarbon receptor and cytochrome P450 1A1 in MCF-7 human breast carcinoma cells. Biochem Pharmacol 56:197-206.

Davies C and Tournier C (2012) Exploring the function of the JNK (c-Jun N-terminal kinase) signalling pathway in physiological and pathological processes to design novel therapeutic strategies. Biochem Soc Trans 40:85-89.

Ding X and Staudinger JL (2005a) Induction of drug metabolism by forskolin: the role of the pregnane $\mathrm{X}$ receptor and the protein kinase a signal transduction pathway. J Pharmacol Exp Ther 312:849-856.
Ding X and Staudinger JL (2005b) Repression of PXR-mediated induction of hepatic CYP3A gene expression by protein kinase C. Biochem Pharmacol 69:867-873.

Dvorak Z, Vrzal R, Henklova P, Jancova P, Anzenbacherova E, Maurel P, Svecova L, Pavek P, Ehrmann J, Havlik R, et al. (2008) JNK inhibitor SP600125 is a partial agonist of human aryl hydrocarbon receptor and induces CYP1A1 and CYP1A2 genes in primary human hepatocytes. Biochem Pharmacol 75:580-588.

Elias A, High AA, Mishra A, Ong SS, Wu J, Peng J, and Chen T (2014) Identification and characterization of phosphorylation sites within the pregnane $\mathrm{X}$ receptor protein. Biochem Pharmacol 87:360-370.

Feng Y, Chambers JW, Iqbal S, Koenig M, Park H, Cherry L, Hernandez P, Figuera-Losada M, and LoGrasso PV (2013) A small molecule bidentate-binding dual inhibitor probe of the LRRK2 and JNK kinases. ACS Chem Biol 8:1747-1754

Ghose R, Guo T, Vallejo JG, and Gandhi A (2011) Differential role of Toll-interleukin 1 receptor domain-containing adaptor protein in Toll-like receptor 2-mediated regulation of gene expression of hepatic cytokines and drug-metabolizing enzymes. Drug Metab Dispos 39:874-881.

Ghose R, Mulder J, von Furstenberg RJ, Thevananther S, Kuipers F, and Karpen SJ (2007) Rosiglitazone attenuates suppression of RXRalpha-dependent gene expression in inflamed liver. J Hepatol 46:115-123.

Ghose R, Zimmerman TL, Thevananther S, and Karpen SJ (2004) Endotoxin leads to rapid subcellular re-localization of hepatic RXRalpha: a novel mechanism for reduced hepatic gene expression in inflammation. Nucl Recept 2:4.

Goey AKL, Meijerman I, Rosing H, Marchetti S, Mergui-Roelvink M, Keessen M, Burgers JA, Beijnen JH, and Schellens JHM (2014) The effect of St John's wort on the pharmacokinetics of docetaxel. Clin Pharmacokinet 53:103-110.

Goodwin B, Redinbo MR, and Kliewer SA (2002) Regulation of cyp3a gene transcription by the pregnane x receptor. Annu Rev Pharmacol Toxicol 42:1-23.

Gripon P, Rumin S, Urban S, Le Seyec J, Glaise D, Cannie I, Guyomard C, Lucas J, Trepo C, and Guguen-Guillouzo C (2002) Infection of a human hepatoma cell line by hepatitis B virus. Proc Natl Acad Sci USA 99:15655-15660.

Guengerich FP (1995) Human cytochrome P-450 enzymes, in Cytochrome P450, 2nd ed (Ortiz de Montellano PR, ed.) pp 450, Plenum, New York.

Guengerich FP (1999) Cytochrome P-450 3A4: regulation and role in drug metabolism. Anпи Rev Pharmacol Toxicol 39:1-17.

Harmsen S, Meijerman I, Beijnen JH, and Schellens JHM (2007) The role of nuclear receptors in pharmacokinetic drug-drug interactions in oncology. Cancer Treat Rev 33:369-380.

Houliston RA, Pearson JD, and Wheeler-Jones CP (2001) Agonist-specific cross talk between ERKs and p38(mapk) regulates PGI(2) synthesis in endothelium. Am J Physiol Cell Physiol 281: C1266-C1276.

Ip YT and Davis RJ (1998) Signal transduction by the c-Jun N-terminal kinase (JNK)-from inflammation to development. Curr Opin Cell Biol 10:205-219.

Jiang W, Welty SE, Couroucli XI, Barrios R, Kondraganti SR, Muthiah K, Yu L, Avery SE, and Moorthy B (2004) Disruption of the Ah receptor gene alters the susceptibility of mice to oxygen-mediated regulation of pulmonary and hepatic cytochromes P4501A expression and exacerbates hyperoxic lung injury. J Pharmacol Exp Ther 310:512-519.

Kim SW, Hasanuzzaman M, Cho M, Heo YR, Ryu MJ, Ha NY, Park HJ, Park HY, and Shin JG (2015) Casein kinase 2 (CK2)-mediated phosphorylation of Hsp90ß as a novel mechanism of rifampin-induced MDR1 expression. J Biol Chem 290:17029-17040.

Kliewer SA, Moore JT, Wade L, Staudinger JL, Watson MA, Jones SA, McKee DD, Oliver BB, Willson TM, Zetterström RH, et al. (1998) An orphan nuclear receptor activated by pregnanes defines a novel steroid signaling pathway. Cell 92:73-82.

Koch P, Gehringer M, and Laufer SA (2015) Inhibitors of c-Jun N-terminal kinases: an update. $J$ Med Chem 58:72-95.

Kodama S and Negishi M (2011) Pregnane X receptor PXR activates the GADD45 $\beta$ gene, eliciting the p38 MAPK signal and cell migration. J Biol Chem 286:3570-3578.

Krishna DR and Klotz U (1994) Extrahepatic metabolism of drugs in humans. Clin Pharmacokine 26:144-160.

Lambert CB, Spire C, Claude N, and Guillouzo A (2009) Dose- and time-dependent effects of phenobarbital on gene expression profiling in human hepatoma HepaRG cells. Toxicol Appl Pharmacol 234:345-360.

Leeder JS and Okey AB (1996) Cytochromes P-450 and liver injury, in Drug Induced Hepatotoxicity pp. 119-153, Springer, Berlin.

Lehmann JM, McKee DD, Watson MA, Willson TM, Moore JT, and Kliewer SA (1998) The human orphan nuclear receptor PXR is activated by compounds that regulate CYP3A4 gene expression and cause drug interactions. J Clin Invest 102:1016-1023.

Lichti-Kaiser K, Brobst D, Xu C, and Staudinger JL (2009a) A systematic analysis of predicted phosphorylation sites within the human pregnane X receptor protein. $J$ Pharmacol Exp Ther 331:65-76.

Lichti-Kaiser K, Xu C, and Staudinger JL (2009b) Cyclic AMP-dependent protein kinase signaling modulates pregnane $\mathrm{x}$ receptor activity in a species-specific manner. J Biol Chem 284 6639-6649.

Liebermann DA and Hoffman B (2008) Gadd45 in stress signaling. J Mol Signal 3:15.

Lin W, Wu J, Dong H, Bouck D, Zeng FY, and Chen T (2008) Cyclin-dependent kinase 2 negatively regulates human pregnane $\mathrm{X}$ receptor-mediated CYP3A4 gene expression in HepG2 liver carcinoma cells. J Biol Chem 283:30650-30657.

Luo G, Guenthner T, Gan L-S, and Humphreys WG (2004) CYP3A4 induction by xenobiotics: biochemistry, experimental methods and impact on drug discovery and development. Curr Drug Metab 5:483-505.

Martínez-Jiménez CP, Jover R, Donato MT, Castell JV, and Gómez-Lechón MJ (2007) Transcriptional regulation and expression of CYP3A4 in hepatocytes. Curr Drug Metab 8:185-194.

Mohammadi-Bardbori A, Bengtsson J, Rannug U, Rannug A, and Wincent E (2012) Quercetin, resveratrol, and curcumin are indirect activators of the aryl hydrocarbon receptor (AHR). Chem Res Toxicol 25:1878-1884

Moore LB, Goodwin B, Jones SA, Wisely GB, Serabjit-Singh CJ, Willson TM, Collins JL, and Kliewer SA (2000) St. John's wort induces hepatic drug metabolism through activation of the pregnane X receptor. Proc Natl Acad Sci USA 97:7500-7502.

Moorthy B, Parker KM, Smith CV, Bend JR, and Welty SE (2000) Potentiation of oxygen-induced lung injury in rats by the mechanism-based cytochrome P-450 inhibitor, 1-aminobenzotriazole. $J$ Pharmacol Exp Ther 292:553-560.

Novotna A and Dvorak Z (2014) Omeprazole and lansoprazole enantiomers induce CYP3A4 in human hepatocytes and cell lines via glucocorticoid receptor and pregnane $\mathrm{X}$ receptor axis. PLoS One 9:e105580. 
Parent R, Marion MJ, Furio L, Trépo C, and Petit MA (2004) Origin and characterization of a human bipotent liver progenitor cell line. Gastroenterology 126:1147-1156.

Pascussi JM, Drocourt L, Gerbal-Chaloin S, Fabre JM, Maurel P, and Vilarem MJ (2001) Dual effect of dexamethasone on CYP3A4 gene expression in human hepatocytes: sequential role of glucocorticoid receptor and pregnane X receptor. Eur J Biochem 268:6346-6358.

Pascussi JM, Gerbal-Chaloin S, Drocourt L, Maurel P, and Vilarem MJ (2003) The expression of CYP2B6, CYP2C9 and CYP3A4 genes: a tangle of networks of nuclear and steroid receptors. Biochim Biophys Acta 1619:243-253.

Pondugula SR, Brimer-Cline C, Wu J, Schuetz EG, Tyagi RK, and Chen T (2009a) A phosphomimetic mutation at threonine-57 abolishes transactivation activity and alters nuclear localization pattern of human pregnane x receptor. Drug Metab Dispos 37:719-730.

Pondugula SR, Dong H, and Chen T (2009b) Phosphorylation and protein-protein interactions in PXR-mediated CYP3A repression. Expert Opin Drug Metab Toxicol 5:861-873.

Rasmussen MK, Daujat-Chavanieu M, and Gerbal-Chaloin S (2017) Activation of the aryl hydrocarbon receptor decreases rifampicin-induced CYP3A4 expression in primary human hepatocytes and HepaRG. Toxicol Lett 277:1-8.

Rochel N, Wurtz JM, Mitschler A, Klaholz B, and Moras D (2000) The crystal structure of the nuclear receptor for vitamin D bound to its natural ligand. Mol Cell 5:173-179.

Shah P, Guo T, Moore DD, and Ghose R (2014) Role of constitutive androstane receptor in Tolllike receptor-mediated regulation of gene expression of hepatic drug-metabolizing enzymes and transporters. Drug Metab Dispos 42:172-181.

Shan E, Zhu Z, He S, Chu D, Ge D, Zhan Y, Liu W, Yang J, and Xiong J (2017) Involvement of pregnane $\mathrm{X}$ receptor in the suppression of carboxylesterases by metformin in vivo and in vitro, mediated by the activation of AMPK and JNK signaling pathway. Eur J Pharm Sci 102:14-23.

Squires EJ, Sueyoshi T, and Negishi M (2004) Cytoplasmic localization of pregnane X receptor and ligand-dependent nuclear translocation in mouse liver. J Biol Chem 279:49307-49314.

Staudinger JL and Lichti K (2008) Cell signaling and nuclear receptors: new opportunities for molecular pharmaceuticals in liver disease. Mol Pharm 5:17-34.

Staudinger JL, Xu C, Biswas A, and Mani S (2011) Post-translational modification of pregnane $\mathrm{x}$ receptor. Pharmacol Res 64:4-10.

Suh HW, Kang S, and Kwon KS (2007) Curcumin attenuates glutamate-induced HT22 cell death by suppressing MAP kinase signaling. Mol Cell Biochem 298:187-194.

Thummel KE and Wilkinson GR (1998) In vitro and in vivo drug interactions involving human CYP3A. Апnи Rev Pharmacol Toxicol 38:389-430.

Usui T, Saitoh Y, and Komada F (2003) Induction of CYP3As in HepG2 cells by several drugs: association between induction of CYP3A4 and expression of glucocorticoid receptor. Biol Pharm Bull 26:510-517.

Vanhooke JL, Benning MM, Bauer CB, Pike JW, and DeLuca HF (2004) Molecular structure of the rat vitamin D receptor ligand binding domain complexed with 2-carbon-substituted vitamin D3 hormone analogues and a LXXLL-containing coactivator peptide. Biochemistry 43:4101-4110.

Veith H, Southall N, Huang R, James T, Fayne D, Artemenko N, Shen M, Inglese J, Austin CP, Lloyd DG, et al. (2009) Comprehensive characterization of cytochrome P450 isozyme selectivity across chemical libraries. Nat Biotechnol 27:1050-1055.
Wakelee HA, Takimoto CH, Lopez-Anaya A, Chu Q, Middleton G, Dunlop D, Ramlau R, Leighl N, Rowinsky EK, Hao D, et al. (2012) The effect of bexarotene on atorvastatin pharmacokinetics: results from a phase I trial of bexarotene plus chemotherapy in patients with advanced non-small cell lung cancer. Cancer Chemother Pharmacol 69:563-571.

Wang Y-M, Ong SS, Chai SC, and Chen T (2012) Role of CAR and PXR in xenobiotic sensing and metabolism. Expert Opin Drug Metab Toxicol 8:803-817.

Waxman DJ, Lapenson DP, Aoyama T, Gelboin HV, Gonzalez FJ, and Korzekwa K (1991) Steroid hormone hydroxylase specificities of eleven cDNA-expressed human cytochrome P450s. Arch Biochem Biophys 290:160-166.

Westerink WMA and Schoonen WGEJ (2007) Phase II enzyme levels in HepG2 cells and cryopreserved primary human hepatocytes and their induction in HepG2 cells. Toxicol In Vitro 21: $1592-1602$.

Wilkinson GR (2005) Drug metabolism and variability among patients in drug response. $N$ Engl J Med 352:2211-2221.

Xie W, Uppal H, Saini SPS, Mu Y, Little JM, Radominska-Pandya A, and Zemaitis MA (2004) Orphan nuclear receptor-mediated xenobiotic regulation in drug metabolism. Drug Discov Today 9(10): $442-449$.

Xu JJ, Diaz D, and O'Brien PJ (2004) Applications of cytotoxicity assays and pre-lethal mechanistic assays for assessment of human hepatotoxicity potential. Chem Biol Interact 150:115-128

Yasunami Y, Hara H, Iwamura T, Kataoka T, and Adachi T (2004) C-jun N-terminal kinase modulates 1,25-dihydroxyvitamin D3-induced cytochrome P450 3A4 gene expression. Drug Metab Dispos 32:685-688.

Zanger UM and Schwab M (2013) Cytochrome P450 enzymes in drug metabolism: regulation of gene expression, enzyme activities, and impact of genetic variation. Pharmacol Ther 138 $103-141$.

Zhang T, Inesta-Vaquera F, Niepel M, Zhang J, Ficarro SB, Machleidt T, Xie T, Marto JA, Kim N, Sim T, et al. (2012) Discovery of potent and selective covalent inhibitors of JNK. Chem Biol 19: 140-154.

Zhou H, Lu N, Chen ZQ, Song QL, Yu HM, and Li XJ (2009) Osteopontin mediates dense cultureinduced proliferation and adhesion of prostate tumour cells: role of protein kinase $\mathrm{C}, \mathrm{p} 38$ mitogen-activated protein kinase and calcium. Basic Clin Pharmacol Toxicol 104:164-170.

Address correspondence to: Dr. Romi Ghose, College of Pharmacy, Department of Pharmacological and Pharmaceutical Sciences, University of Houston, 1441 Moursund Street, Houston, TX 77030. E-mail: rghose@central.uh.edu; or Dr. Bhagavatula Moorthy, Department of Pediatrics, Baylor College of Medicine, 1102 Bates Avenue, Suite 530, Houston, TX 77030. E-mail: bmoorthy@bcm.tmc. edu 